\title{
Multi-scale community organization of the human structural connectome and its relationship with resting-state functional connectivity
}

\author{
RICHARD F. BETZEL \\ Department of Psychological and Brain Sciences, Indiana University, Bloomington, IN, USA \\ Program in Cognitive Science, Indiana University, Bloomington, IN, USA \\ ALESSANDRA GRIFFA \\ Department of Radiology, University Hospital Center and University of Lausanne (CHUV), \\ Lausanne, Switzerland \\ Signal Processing Laboratory (LTS5), Ecole Polytechnique Fédérale de Lausanne (EPFL), \\ Lausanne, Switzerland
}

ANDREA AVENA-KOENIGSBERGER

Department of Psychological and Brain Sciences, Indiana University, Bloomington, IN, USA Program in Cognitive Science, Indiana University, Bloomington, IN, USA

\section{J O A Q UÍN GOÑ I}

Department of Psychological and Brain Sciences, Indiana University, Bloomington, IN, USA

\author{
JEAN-PHILIPPE THIRAN and PATRIC HAGMANN \\ Department of Radiology, University Hospital Center and University of Lausanne (CHUV), \\ Lausanne, Switzerland \\ Signal Processing Laboratory (LTS5), Ecole Polytechnique Fédérale de Lausanne (EPFL), \\ Lausanne, Switzerland

\section{OLAF SPORNS}

Department of Psychological and Brain Sciences, Indiana University, Bloomington, IN, USA Program in Cognitive Science, Indiana University, Bloomington, IN, USA

(e-mail: osporns@indiana.edu)

\begin{abstract}
The human connectome has been widely studied over the past decade. A principal finding is that it can be decomposed into communities of densely interconnected brain regions. Past studies have often used single-scale modularity measures in order to infer the connectome's community structure, possibly overlooking interesting structure at other organizational scales. In this report, we used the partition stability framework, which defines communities in terms of a Markov process (random walk), to infer the connectome's multi-scale community structure. Comparing the community structure to observed resting-state functional connectivity revealed communities across a broad range of scales that were closely related to functional connectivity. This result suggests a mapping between communities in structural networks, models of influence-spreading and diffusion, and brain function. It further suggests that the spread of influence among brain regions may not be limited to a single characteristic scale.
\end{abstract}

Keywords: connectome, community structure, multi-scale, Markov process, resting-state 


\section{Introduction}

Many complex networks exhibit community structure, defined for example by clustered edge distributions such that vertices (nodes) in the same community preferentially link to one another (Guimera \& Amaral, 2005; Girvan \& Newman, 2002; Newman \& Girvan, 2004). Examples of community structure can be found in society as groups of friends, workplaces, cities, and states (Moody \& White, 2003; Freeman, 2004); in protein interaction networks as groups of co-functioning proteins (Jonsson et al., 2006); and in the World Wide Web (WWW) as webpages sharing many hyperlinks (Albert et al., 1999; Flake et al., 2002).

Detecting community structure is an important endeavor in network science. Though there exist many methods for doing so, none has emerged as clearly preeminent (for a review, see Fortunato, 2010). One of the most widely used methods is to identify the vertex partition that maximizes the modularity quality function, defined as the difference between observed and expected intra-community edge density (Newman, 2006). Despite its widespread usage, modularity analysis suffers from several drawbacks: maximization is NP-hard and identifying the optimal partition is often practically impossible (Fortunato, 2010); solutions are sometimes degenerate, with multiple partitions corresponding to the maximum modularity (Good et al., 2010); and a resolution limit renders modularity "blind" to communities below some characteristic scale (Fortunato \& Barthelemy, 2007).

The first two problems are ubiquitous to most community detection algorithms and are generally unavoidable. The resolution limit is more specific to quality functions like modularity, but has been remedied in several instances by the inclusion of a tunable resolution parameter, which controls the scale at which communities are detected (Reichardt \& Bornholdt, 2006; Arenas et al., 2008; Ronhovde \& Nussinov, 2009) and has been generalized to include multi-slice networks (Mucha et al., 2010). In neuroscience, multi-slice community detection has been studied in the context of alterations in the community structure of functional brain networks over the course of learning (Bassett et al., 2011). Outside of neuroscience, multi-scale/slice community detection has been applied in fields including economics (Fenn et al., 2009, 2012), protein interaction networks (Lewis et al., 2010; Delmotte et al., 2011), and other examples of social and biological networks (Mucha et al., 2010; Onnela et al., 2012).

This manuscript focuses on a particular multi-scale community detection algorithm known as the partition stability framework, which defines communities in terms of a Markov process based on a random walk model (Lambiotte et al., 2008; Delvenne et al., 2010; Lambiotte, 2010). As this process evolves, a random walker makes progressively longer walks and explores more distant parts of the network. Intuitively, communities can be thought of as groups of vertices that effectively "trap" the flow of random walkers over a particular timescale of the random walk. In this way, the stability framework has been dubbed a "zooming lens," whereby focusing in on shorter or longer timescales reveals communities of correspondingly smaller or larger diameter (Schaub et al., 2012). This method can be mapped to the methods proposed by Reichardt \& Bornholdt (2006) and Arenas et al. (2008), indicating a mathematical equivalence. The partition stability framework, however, differs conceptually from the other methods in a subtle wayits resolution parameter (time) can be interpreted in the context of a random walk 
process, whereas the resolution parameters of Reichard \& Bornholdt (2006) and Arenas et al. (2008) were introduced $a d$ hoc and have no additional interpretation.

The human connectome, i.e. the full set of neural elements and connections of the human brain, can be modeled as a complex network (Sporns et al., 2005; Bullmore $\&$ Sporns, 2009). The topological properties of this network have been studied for nearly a decade, revealing key features including small-world architecture (Gong et al., 2009), hub regions and cores (Hagmann et al., 2008), rich club organization (van den Heuvel \& Sporns, 2011), modular architecture (Chen et al., 2008; Meunier et al., 2010; Wu et al., 2011), and economical wiring (Bassett et al., 2010; Bullmore $\&$ Sporns, 2012). While these results characterize and contextualize the connectome among all complex networks, the role of these topological features in shaping communication processes and dynamic couplings among brain regions remains an area of active research (Honey et al., 2009; van den Heuvel et al., 2012; Haimovici et al., 2013).

Empirical and computational studies suggest that the human connectome underpins complex neural dynamics and facilitates the communication and integration of information between brain regions. In functional magnetic resonance imaging (fMRI), such "functional connectivity" is reflected by the magnitude of statistical dependence, commonly measured as linear correlation, between blood oxygen-level dependent (BOLD) signals recorded from different brain regions. A growing body of literature describes patterns of resting-state functional connectivity ( $\mathrm{rsFC}$ ), i.e. spontaneous, endogenous fluctuations of the BOLD signal in the absence of any explicit cognitive task. This work has revealed consistent connectivity patterns, dubbed resting-state networks (RSN), which resemble networks of regions (e.g., somato-motor, visual, default mode, etc.) that are coherently engaged in various cognitive and behavioral domains (Greicius et al., 2003; Damoiseaux et al., 2006; Fox \& Raichle, 2007; Smith et al., 2009).

This article has two principal aims. The first aim consists of detecting and characterizing the multi-scale community structure of the human connectome using the partition stability framework. The second aim is to assess the relationship of such structure to empirically observed rsFC. Where the first aim attempts to answer the question "what communities of brain regions are situated in such a way that they could take advantage of a diffusion-like process in order to pass information or spread influence among themselves," the second aim attempts to answer the question "what evidence is there that the previously-identified communities match observed patterns of functional connectivity and at which scales is the correspondence between community structure and functional coupling most salient?"

\section{Methods}

\subsection{Neuroimaging and data acquisition}

Forty (40) healthy human volunteers ( 24 males and 16 females, $25.3 \pm 4.9$ years old) underwent an MRI session on a 3-Tesla scanner (Trio, Siemens Medical, Germany) with a 32-channel head-coil. The session consisted of (i) a magnetization-prepared rapid acquisition gradient echo (MPRAGE) sequence sensitive to white/gray matter contrast (1 mm in-plane resolution, $1.2 \mathrm{~mm}$ slice thickness), (ii) a diffusion spectrum imaging (DSI) sequence (128 diffusion weighted volumes +1 reference $b_{0}$ volume, 
maximum $b$-value $8000 \mathrm{~s} / \mathrm{mm}^{2}, 2.2 \times 2.2 \times 3.0 \mathrm{~mm}$ voxel size), and (iii) a gradient echo EPI sequence sensitive to BOLD contrast $(3.3 \mathrm{~mm}$ in-plane resolution and slice thickness with a $0.3 \mathrm{~mm}$ gap, TR $1920 \mathrm{~ms}$ ). During the fMRI acquisition, subjects were lying in the scanner with eyes open, resting but awake and cognitively alert, thus recording-resting-state fMRI (rs-fMRI). The acquisition process resulted in a sequence of 276 BOLD images for each subject.

DSI, rs-fMRI, and MPRAGE data were processed using the Connectome Mapping Toolkit (Daducci et al., 2012). Each participant's gray and white matter compartments were segmented from the MPRAGE volume. The entire cortical and subcortical volume was subdivided into 1015 equal sized regions of interest (Cammoun et al., 2012). The present study, however, focused on cortical structures only, and discarded subcortical regions including the bilateral thalamus, caudate, putamen, pallidum, accumbens, hippocampus, and amygdala, as well as the brainstem, resulting in 1000 cortical regions of interest. Each region, in turn, could be mapped to one of 68 cortical areas, with 34 areas per hemisphere (for a complete list of cortical areas and their mapping to regions of interest, see the Supplementary Information). Whole brain streamline tractography was performed on reconstructed DSI data (Wedeen et al., 2008), and participant-wise, right hemisphere connectivity matrices were estimated by selecting the streamlines connecting each pair of 501 cortical regions in the right hemisphere. Connectivity strength between each pair of regions was quantified as fiber density, which is defined as the number of streamlines connecting the two regions, normalized by the average streamline's length, and the average surface of the two connected areas (Hagmann et al., 2008). The normalization by the average streamline's length is meant to compensate for the bias toward longer fibers introduced by the tractography algorithm. The connectivity values quantified as fiber density were used to construct subject-wise structural connectivity (SC) matrices.

Each SC matrix can be interpreted as the adjacency matrix $A_{i j}$ of a graph $G \equiv\{V, E\}$, with vertices $V=\left\{v_{1}, \ldots, v_{n}\right\}$ corresponding to cortical regions of interest, and weighted, undirected edges $E=\left\{e_{i j}, \ldots, e_{k l}\right\}$ representing anatomical connections. This network is often referred to as a structural connectome. It is convenient at this point to define a connectome's vertex strength and total weight as $s_{i}=\Sigma_{j} A_{i j}$ and $m=\frac{1}{2} \Sigma_{i j} A_{i j}$, respectively.

Functional data were pre-processed according to state of the art pipelines (Murphy et al., 2009; Power et al., 2012). The raw fMRI volumes were motion-corrected by applying rigid body co-registration. Voxel-wise signals were then corrected for physiological confounds and artifacts by regressing out the average white matter signal, the average cerebrospinal fluid signal, and the six motion signals (three translations and three rotations) previously estimated. The time series were lowpass filtered (temporal Gaussian filter with full width at half maximum $=1.92 \mathrm{~s}$ ), and the first four time points were excluded to allow signal stabilization. No spatial smoothing was applied. Following "motion scrubbing" (Power et al., 2012), voxel-wise fMRI signals were averaged across each cortical region in order to obtain a representative time series for each region. The functional connectivity between each pair of regions was estimated as the Pearson correlation between the corresponding average signals. A single, right hemisphere rsFC matrix $F_{i j}$ representative of the whole group of participants was computed by averaging the 40 individual correlation matrices. The group-mean structural connectome (weighted and binary) and functional connectivity matrix for the right hemisphere of cerebral 

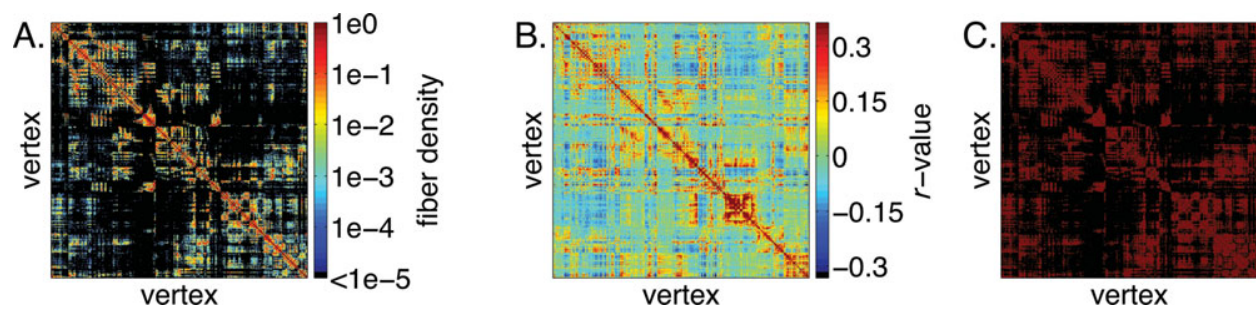

Fig. 1. Structural and functional matrices averaged over 40 participants; (A) SC weighted: entries indicate the average density of white matter fiber tracts connecting brain regions; (B) SC binary: entries indicate the average presence(absence) of connecting fibers present in any of the 40 participants; (C) rsFC: entries are averaged correlations of the processed BOLD time series during resting state. (Color online)

cortex are shown in Figure 1. Each participant's weighted SC matrix was generated following the procedure described above, while binary matrices were generated by setting each nonzero element in the weighted SC matrix equal to unity. The group-mean SC matrices were then generated by averaging weighted and binary SC matrices across all participants.

The decision to focus solely on the right hemisphere was based on the strong symmetry of the right and left hemispheres in terms of both structural and functional networks, greater uncertainty about inference of inter-hemispheric structural connections, as well as previous analyses conducted only on single hemisphere brain networks (e.g., Vèrtes et al., 2012).

The decision to aggregate each of the 40 participants' rsFC matrices into a group average matrix also requires some justification. There were two reasons for doing so: (i) the aim of this study was not to categorically identify and study the individual differences between subjects. Instead, the aim was to find commonalities among all the participants; (ii) each resting state run consisted of 276 samples $(\approx 9$ minutes), which may be an insufficient number of samples to fully characterize the long-time covariance structure of BOLD activity in a single subject (Birn et al., 2013). However, assuming that the true underlying covariance structure is similar across subjects, then averaging rsFC matrices gives us a more stable estimate of that structure. As a test of how similar the group average rsFC was to the individual matrices, the upper triangle of each single subject matrix was converted into a vector along with the group average matrix, and the correlations of the group average vector and the single-subject vector were computed. The median correlation coefficient was $r=0.509$ and the minimum and maximum correlations were 0.356 and 0.570 , respectively.

\subsection{Partition stability framework}

One of the main aims of this article was to detect the connectome's multi-scale community structure using the partition stability framework. This framework defines communities in terms of a Markov process - in this article, a continuous-time random walk. Such a process can also be used to model the diffusion of information or energy over a network, and is defined by the dynamical system:

$$
\dot{p}_{i}=-\sum_{j} L_{i j} p_{j}
$$


where $p_{i}$ is the probability of finding a random walker on vertex $v_{i}$ and $L_{i j}=$ $\delta_{i j}-A_{i j} / s_{j}$ is the normalized graph Laplacian matrix. If the network is undirected and connected, this system evolves to the equilibrium state $p_{i}^{*}=s_{i} / 2 \mathrm{~m}$.

The stability framework detects community structure at different timescales of the random walk (referred to simply as "scales" so as to avoid any confusion with time in the context of intrinsic brain dynamics). The communities were detected by identifying the vertex partition that maximizes the quality function "stability." Let $\wp=\left\{\mathscr{C}_{1}, \ldots, \mathscr{C}_{K}\right\}$ be a partition of $V$ into $K$ communities, such that $\mathscr{C}_{i} \cap_{j}^{\mathscr{C}}=\emptyset$ and $\cup_{i} \mathscr{C}_{i}=V$. The stability of $\wp$ at a given scale $t$ is defined as:

$$
R(\wp, t)=\sum_{\mathscr{C} \in \wp} \sum_{i j \in \mathscr{C}}\left[\left(e^{-t L}\right)_{i j} p_{j}^{*}-p_{i}^{*} p_{j}^{*}\right]
$$

where the summation extends over all communities and the edges that fall within each community. The first term in the summation, $\left(e^{-t L}\right)_{i j} p_{j}^{*}$, is the probability that a random walker starting in community $\mathscr{C}$ will be in that community at scale $t$. The second term $p_{i}^{*} p_{j}^{*}$ is the probability that two independent random walkers will be in $\mathscr{C}$ at equilibrium. The difference in these terms represents the density of random walkers in a community in excess of what is expected at equilibrium. Key to this framework, the stability measurement depends not only on the partition $\wp$, but also on the scale $t$. In general, different partitions will maximize stability at different scales in the random walk. Varying $t$ across scales and maximizing stability recovers the community structure at each scale.

The process of optimizing $R(\wp, t)$ can be accomplished in a number of ways. One appealing option, and the one used in this article, makes use of the relationship between partition stability and a more widely used modularity measure. Lambiotte et al. $(2008,2011)$ demonstrated that stability could be recast in terms of the modularity of a weighted, symmetric "flow graph." A flow graph is a transformation of $A_{i j}$ in which the dynamics of a Markov process are embedded into the edges of a new graph. In the case of a continuous-time random walk, the flow graph is represented by the full matrix $A_{i j}^{\prime}(t)=\left(e^{-t L}\right)_{i j} s_{j}$ whose elements are proportional to the probabilistic flow of random walkers between vertices at scale $t$. Examples of flow graphs for one of the participants are shown in Figure 2. The relationship between stability and modularity is such that instead of directly maximizing $R(\wp, t)$, one can equivalently maximize the modularity of a flow graph evaluated at scale $t$ :

$$
Q(\wp, t)=\frac{1}{2 m} \sum_{\mathscr{C} \in \wp} \sum_{i j \in \mathscr{C}}\left[A_{i j}^{\prime}(t)-\frac{s_{i} s_{j}}{2 m}\right]
$$

This result confers the practical advantage that any heuristic previously used to maximize modularity can now be repurposed in order to maximize stability.

To apply this set of principles to the connectomes obtained from 40 healthy individuals, a range of scales had to be selected over which to maximize stability. After experimentation, it was determined that at scales below $t=10^{-3.5}$, every participant's community structure was characterized by a partition into $n$ communities, i.e. every vertex was assigned to its own community, and at scales greater than $t=10^{3.5}$ every participant exhibited a two-community division. Using these scales as lower and upper boundaries, we selected 185 logarithmically spaced points over the interval $\left[10^{-3.5}, 10^{3.5}\right]$ at which to maximize partition stability. This process entailed defining a flow graph $A_{i j}^{\prime}(t)$ for each participant, evaluating that flow graph at each of the 

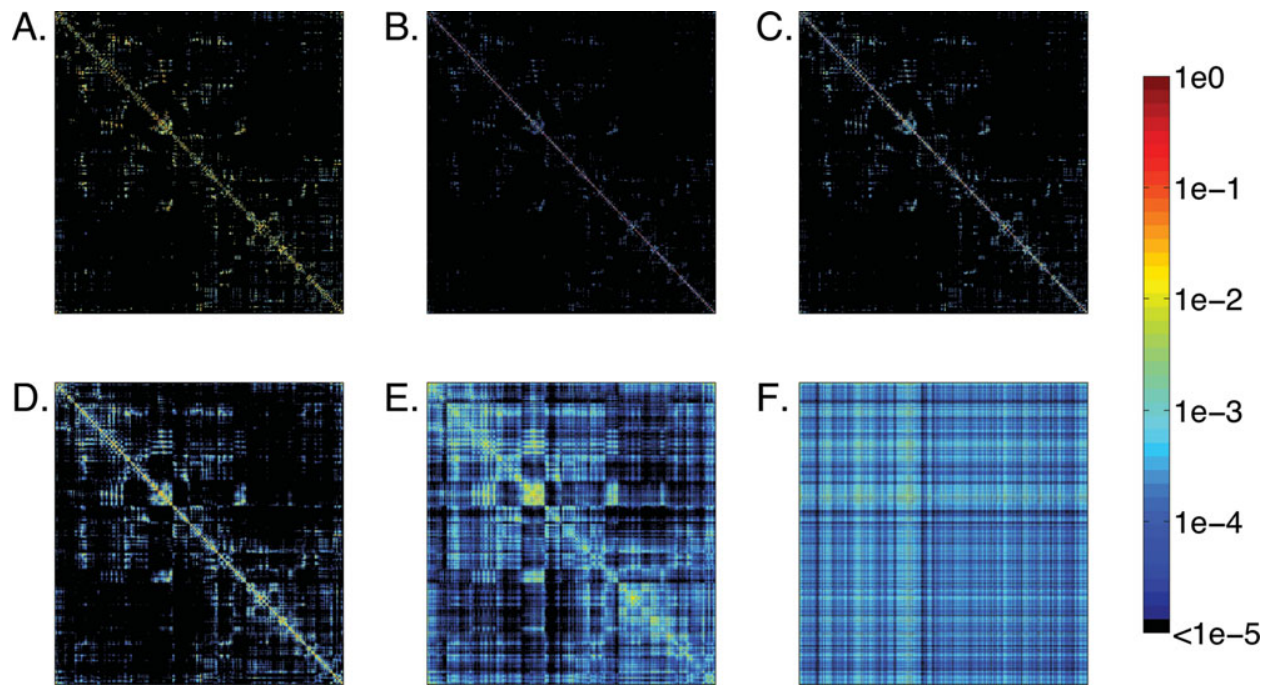

Fig. 2. Examples of flow graphs obtained at different Markov times in a single participant; (A) original SC matrix; (B)-(F) flow graphs at times $10^{-2}, 10^{-1}, 10^{0}, 10^{+1}, 10^{+2}$, respectively, depicting the probabilistic flow of random walkers between pairs of vertices. (Color online)

pre-selected scales, and subsequently maximizing each flow graph's modularity (or equivalently, stability) by applying the Louvain algorithm 750 times (Blondel et al., 2008). At a given scale, a participant's community structure was represented by the partition corresponding to the maximum modularity (stability).

Rather than analyze the multi-scale community structure of each participant individually, communities were aggregated across all participants to shift focus onto the group-average community structure. This operation was summarized by the weighted, symmetric and scale-dependent agreement matrix $D_{i j}(t)$, whose elements indicated the percentage of all participants in which, at scale $t$, vertices $v_{i}$ and $v_{j}$ were assigned to the same community. Thus, the elements of $D_{i j}(t)$ were interpreted as the probability that two vertices belonged to the same community. Values ranged from " 0 " in the case of two vertices that never appeared in the same community, to " 1 " for two vertices that always appeared in the same community across all participants. Conceptually, $D_{i j}(t)$ reflected the extent to which participants' community structures at each scale coincided with one another.

The agreement matrix $D_{i j}(t)$ proffered a probabilistic description of the connectome's community structure as a function of scale. It was of practical interest to obtain at each scale a single partition corresponding to the connectome's consensus communities, i.e. the communities that were common to the majority of participants. To obtain such community structure, an iterative thresholding/clustering algorithm was applied to $D_{i j}(t)$ (Lancichinetti \& Fortunato, 2012). This procedure consisted of two steps: (i) All edges in $D_{i j}(t)$ below a threshold $\tau=0.5$ were regarded as "noise" and were set to zero. Imposing such a threshold resulted in the matrix $D_{i j}^{\prime}(t)$ whose remaining edges linked only those vertices assigned to the same community greater than half of the time; (ii) $D_{i j}^{\prime}(t)$ was then clustered 100 times using the Louvain algorithm (Blondel et al., 2008). If the resulting partitions were identical, then the algorithm had reached consensus and it terminated. Otherwise, a metaagreement matrix was built from the new partitions and the algorithm returned 
to (i). At every scale, then, this procedure found a consensus partition $\wp(t)$ that captured only the features of community structure common to the majority of participants and ignored the features unique to individuals. The ordered set of consensus partitions was summarized as $\Phi=\left\{\wp\left(t=10^{-3.5}\right), \ldots, \wp\left(t=10^{3.5}\right)\right\}$. It should be noted that, in general, the process of obtaining a consensus partition resulted in a loss of information, removing the features of community structure that were expressed infrequently in participants. While such information might reveal meaningful differences between subjects, it was disregarded in all further analyses, which focus on characterizing features of community structure common among the cohort of participants.

The choice to set $\tau=0.5$ was made following some experimentation and after considering several alternative values. First, values of $\tau$ ranging from 0 to 1 in increments of 0.1 were used to compute consensus communities at select scales. This experimentation confirmed that when $\tau$ is set to an extreme value, i.e. close to 0 or 1 , the consensus partition tends to over- or under-cluster the data, resulting in either fewer or greater communities than the number exhibited by any single participant.

Another attractive option was to use a data-driven approach, whereby $\tau$ is automatically chosen based on some statistic of the partitions being considered. One appealing option was proposed by Bassett et al. (2013) and entailed randomly permuting the community assignments of the individual participants' partitions (many times) and computing a second agreement matrix from the randomized partitions to test the hypothesis that two vertices were assigned to the same community by chance. The authors of that paper suggested setting $\tau$ equal to the maximum value in the null agreement matrix. In the present study, however, adopting such a heuristic produced values for $\tau$ close to 0 , which tended to fall into the regime of parameter space that generated over-clustered consensus partitions. Furthermore, the low $\tau$ values meant that this procedure often took multiple iterations to reach consensus, effectively increasing the number of steps between the consensus partition and the input partitions, and thus inflating the possibility that the consensus partition might become dissimilar to the individual participant's partition.

Given that extreme values of $\tau$ appeared to be a poor choice and that, though initially promising, the data-driven approach produced poor results, $\tau$ was set equal to 0.5 as a compromise. It should be noted, however, that there are other methods available for selecting consensus clusters (Strehl \& Ghosh, 2003; Topchy et al., 2005; Yu et al., 2007; Carpineto \& Romano, 2012; Chu et al., 2012). Comparing the outcomes of different consensus clustering techniques fell beyond the stated aims of this article.

\subsection{Relating community structure to functional connectivity}

A major aim of this article was to gain insight into how the connectome's multiscale community structure related to brain function. To map this relationship, we compared the agreement matrix $D_{i j}(t)$ and the sequence of consensus partitions $\Phi$ to the empirically measured $\mathrm{rsFC}$ matrix $F_{i j}$.

First, as a measure of correspondence between $\mathrm{rsFC}$ and the consistency of community structure across subjects, we computed the Pearson correlation of the upper-triangle elements in the empirically measured $\operatorname{rsFC}$ matrix $F_{i j}$ with $D_{i j}(t)$ at 
each scale $t$. Larger correlation values implied that the propensity for two vertices to share a community assignment was a good predictor of whether those same vertices were also functionally coupled. It should be noted that this measurement does not establish a direct link between community structure and rsFC, but instead links the reliability of community structure to rsFC.

As a second measure of correspondence, each consensus community's "goodness" was determined by imposing it upon the rsFC matrix $F_{i j}$. Conceptually, a "good" community was one whose internal density of positive functional connections and external negative connections were greater than expected by chance. This intuition of a community's "goodness" was in line with definitions of modularity adopted for use with signed networks (Traag \& Bruggeman, 2009; Rubinov \& Sporns, 2011). Therefore, a consensus community's "goodness" with respect to rsFC was estimated by computing its modularity score. The modularity of a community in a signed network is defined as:

$$
q_{\mathscr{C}}^{*}=\frac{1}{2 m^{+}} \sum_{i j \in \mathscr{C}}\left[F_{i j}^{+}-\frac{s_{i} s_{j}}{2 m^{+}}\right]-\frac{1}{2 m^{+}+2 m^{-}} \sum_{i j \in \mathscr{C}}\left[F_{i j}^{-}-\frac{s_{i}^{-} s_{j}^{-}}{2 m^{-}}\right]
$$

where $F_{i j}^{ \pm}$are rsFC matrices comprised of only positive $(+)$and negative $(-)$ connections, $s_{i}^{ \pm}=\sum_{j} F_{i j}^{ \pm}$is the signed vertex strengths, and $m^{ \pm}=\frac{1}{2} \sum_{i j} F_{i j}^{ \pm}$is the total signed weight of the network.

Large communities, because they consisted of many vertices, also tended to have large modularity scores. To remove this bias, the procedure described above was repeated 5,000 times but with vertices randomly assigned to communities $\mathscr{C}_{\text {rand }}$, each time resulting in a measurement $q_{\mathscr{C}_{\text {rand }}}^{*}$. This score enabled estimates of the expected value $\left(E\left[q_{\mathscr{C}_{\text {rand }}}^{*}\right]\right)$ and variance $\left(\sigma\left[q_{\mathscr{C}_{\text {rand }}}^{*}\right]\right)$ of each community's modularity score to be obtained. From these estimates, a community's score was standardized and expressed as a $z$-score:

$$
z_{\mathscr{C}}^{*}=\frac{\left(q_{\mathscr{C}}^{*}-E\left[q_{\mathscr{C}_{\text {rand }}}^{*}\right]\right)}{\sigma\left[q_{\mathscr{C}_{\text {rand }}}^{*}\right]}
$$

The score $z_{\mathscr{C}}$ was interpreted as an indicator of how well each structural community $\mathscr{C} \in \wp(t)$ mapped onto rsFC. A large, positive $z_{\mathscr{C}}$ indicated that a community's signed modularity was much greater than would be expected given its size.

In addition to identifying communities that were more modular than by chance, the scores $z_{\mathscr{C}}$ were useful for answering several important questions about how stability-derived communities related to rsFC: (i) On average which cortical areas contributed the most standardized modularity; (ii) which pairs of vertices; and (iii) which pairs of cortical areas, when assigned to the same community, portended a large standardized modularity score for that community.

From the set of standardized modularity scores, it was straightforward to compute the total standardized modularity contribution of each cortical area by summing the scores of each vertex $v_{i}$ over all communities in which $v_{i}$ participated and then aggregating these scores by cortical area.

Another important question was how the co-assignment of groups of vertices or cortical areas to a given community influenced that community's modularity. For example, does assigning vertices $v_{i}$ and $v_{j}$ to the same community portend a higher or lower modularity for that community? To identify such groups, a matrix $T_{i j}$ 
was built and subsequently clustered. Initially, the weights of $T_{i j}$ were set to zero. The weights were updated by considering each community $\mathscr{C}$ and strengthening the connections among all of the vertices assigned to $\mathscr{C}$ by $z_{\mathscr{C}}$. For example, suppose a community $\mathscr{C}$ was comprised of vertices $1-10$ and that this community's previously measured standardized modularity score was $z_{\mathscr{C}}=3$. Were this the case, then the elements $T_{i j}$ for $i, j=1, \ldots, 10$ would be uniformly increased by $z_{\mathscr{C}}=3$. This process would be repeated for each community in the set of all communities, $\Phi$. Thus, the weights of $T_{i j}$ were equal to the sum of standardized modularity over all communities in which vertices $v_{i}$ and $v_{j}$ both appeared. An agglomerative, hierarchical clustering algorithm was then used to extract groups of vertices that collectively influenced a community's modularity (Hastie et al., 2001). This algorithm treated each row in $T_{i j}$ as a feature vector of the matching vertex $v_{i}$. Starting with every vertex in its own cluster, clusters were merged over a series of steps until only two clusters remained. At each step, the relationship between every pair of clusters was defined by the average Euclidean distance between the feature vectors of vertices assigned to those clusters. The heuristic for merging clusters was to identify the two clusters whose distance was smallest and to combine their elements, forming a larger joint cluster at the next step. This procedure produced a hierarchical tree of related vertices which can be thresholded, revealing a finer or coarser clustering depending on the level of the threshold. At any level, however, these clusters were interpreted as groups of vertices that collectively participated in communities with large standardized modularity scores. To identify pairs of cortical areas whose coassignment contributed to a community's having small or large modularity, $T_{i j}$ was down-sampled by aggregating its rows and columns according to cortical areas.

\section{Results}

The previous section described a procedure for identifying the connectome's multiscale community structure using the partition stability framework. The association between this structure and observed functional connectivity was assessed by correlating it with rsFC and, separately, by measuring how well consensus communities modularized the rsFC matrix.

Maximizing partition stability at 185 different scales logarithmically spaced over the range $\left[10^{-3.5}, 10^{+3.5}\right]$ generated a sequence of partitions for each participant. Each sequence began at the shortest dynamical scale with the partition in which every vertex comprised its own community. A division of the vertex set into two communities characterized the final partition in each sequence, corresponding to the largest scale. From these partition sequences, a number of statistics were computed at each scale, specifically, the mean and standard deviation of the partition stability, number of communities, community size, and number of singleton communities. Partition stability and the number of communities declined monotonically while the size of communities increased. The number of singleton communities declined initially, before the maximum number of non-singleton communities reached a maximum value at the scale $t=10^{-1.845}$ (Figure 3).

An iterative thresholding/reclustering algorithm was used to generate from the participants' partitions a sequence of consensus partitions, which represented the "backbone" community structure. Typically, this procedure required only one 


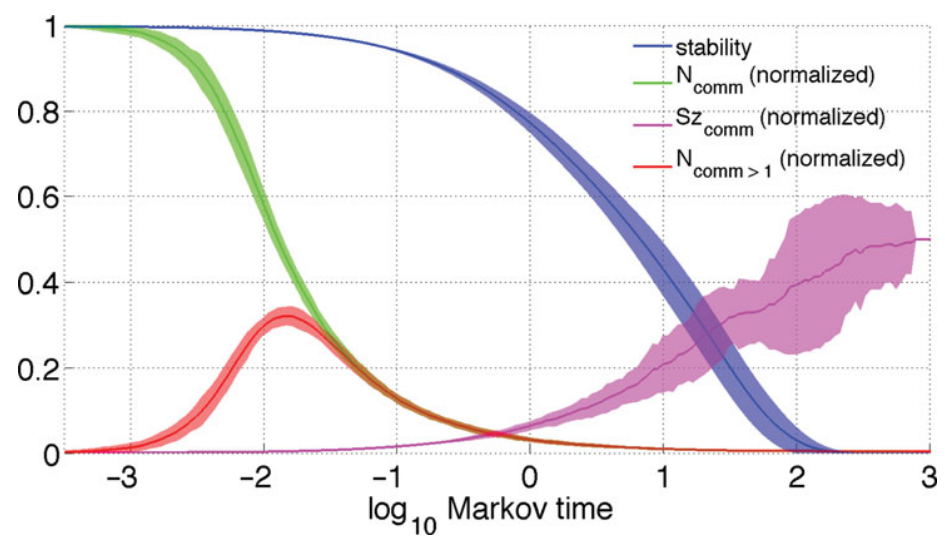

Fig. 3. Statistics from community structure obtained from stability optimization procedure (all curves depict mean plus/minus two standard deviations); partition stability (blue); number of communities (green); number of communities with more than one vertex (red); average community size (magenta). Community size, number of singleton communities, and number of communities are normalized by size of the network (501 cortical regions in the right hemisphere) so that they scale between 0 and 1 . (Color online)

A.

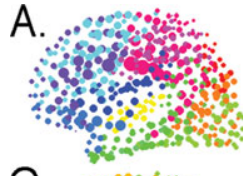

C.

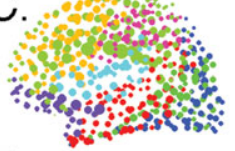

E.

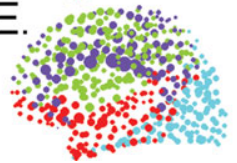

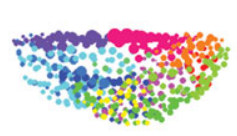
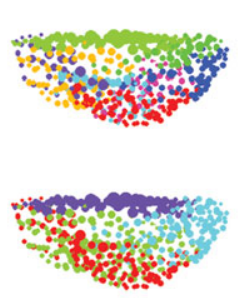
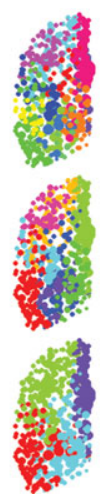

B.

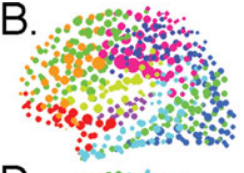

D.
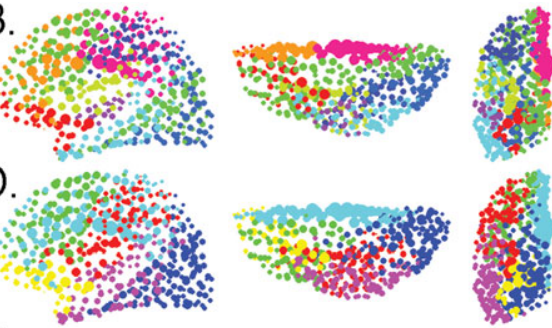

F.

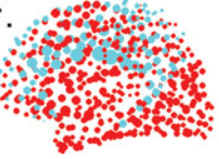

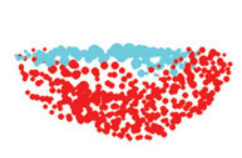

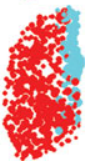

Fig. 4. Consensus partitions obtained at different Markov times; $(\mathrm{A}) t \approx 10^{-0.23}$ with 12 communities; (B) $t \approx 10^{0.08}$ with 10 communities; (C) $t \approx 10^{0.273}$ with eight communities; (D) $t \approx 10^{0.53}$ with six communities; (E) $t \approx 10^{0.76}$ with four communities; $(\mathrm{F}) t \approx 10^{2.89}$ with two communities. Vertex size is proportional to strength. (Color online)

iteration (and never more than two) before achieving consensus. Examples of consensus partitions at selected scales are shown in Figure 4(A)-(F). Each partition represents a division of the cerebral cortex into communities of vertices that tended to "trap" the flow of random walkers over a given scale. (For a list of all vertices and their community assignments at each scale, see the Supplementary Information.)

To compare community structure to brain functional connectivity, the Pearson correlation of the scale-dependent agreement matrix $D_{i j}(t)$ with the empirically measured rsFC matrix $F_{i j}$ was computed, peaking at a value of $r=0.50$ at scale $t=10^{-0.455}$ (Figure 5). This level of correlation is comparable to earlier studies reporting correspondence between the structural connectome and rsFC, as well as correlations between functional connectivity generated in computational models and empirical rsFC (Honey et al., 2009). An interesting observation was that the 

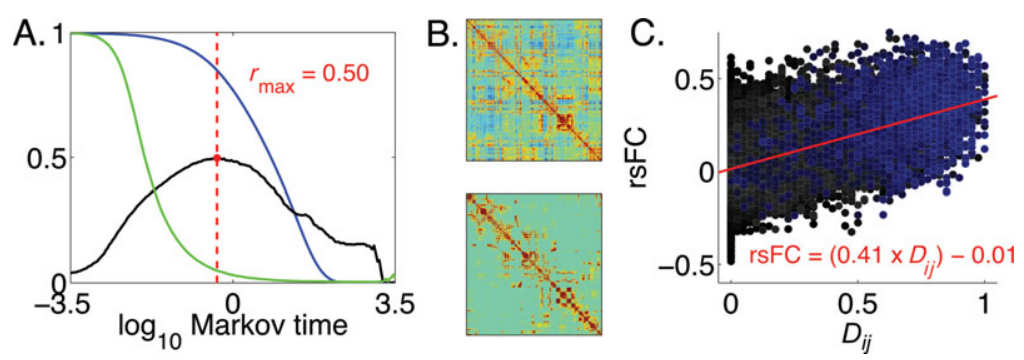

Fig. 5. Summary of correlation between agreement matrix $D_{i j}(t)$ and resting-state functional connectivity (rsFC); (A) Pearson's correlation value (black) at each time point. Superimposed on this plot are the mean stability (blue) and mean number of community (green) curves. The time at which the max correlation value occurs falls within a narrow regime where the number of communities and stability are both substantial; (B) $D_{i j}(t)$ at peak correlation value (top) and rsFC (bottom); (C) scatter plot of $D_{i j}(t)$ and rsFC at time of peak correlation (linear fit-red line). The color of each "o" denotes the number of participants in which the corresponding structural connection was also present. (Color online)
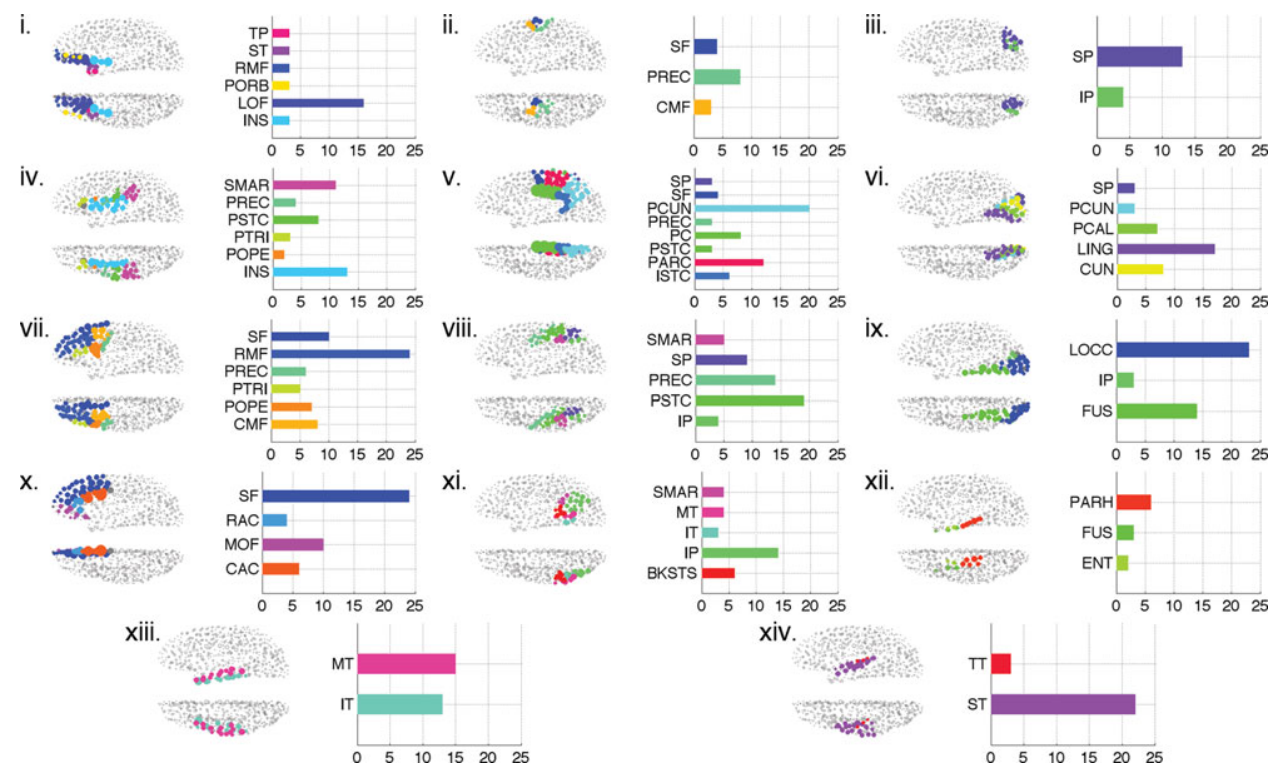

Fig. 6. Summary of 14 consensus communities at the time of peak correlation. In each plot, vertices are colored gray if they do not belong to the corresponding community. The color of any non-gray vertex indicates the cortical region that the vertex belongs to. Once again, vertex size is proportional to strength. (Color online)

peak correlation occurred at a scale where there were many communities made up of many vertices $(24.53 \pm 2.06$ communities with an average $20.65 \pm 1.69$ vertices per community) whose stability $(0.85 \pm 0.01)$ had not yet begun to decay. Earlier scales had slightly greater stability values but were characterized by having far more singleton communities, while partitions at later Markov times had no singleton communities but were marked by extremely low stability values. The consensus partition at this time consisted of 14 communities, whose topographical arrangement and composition are shown in Figure 6. 

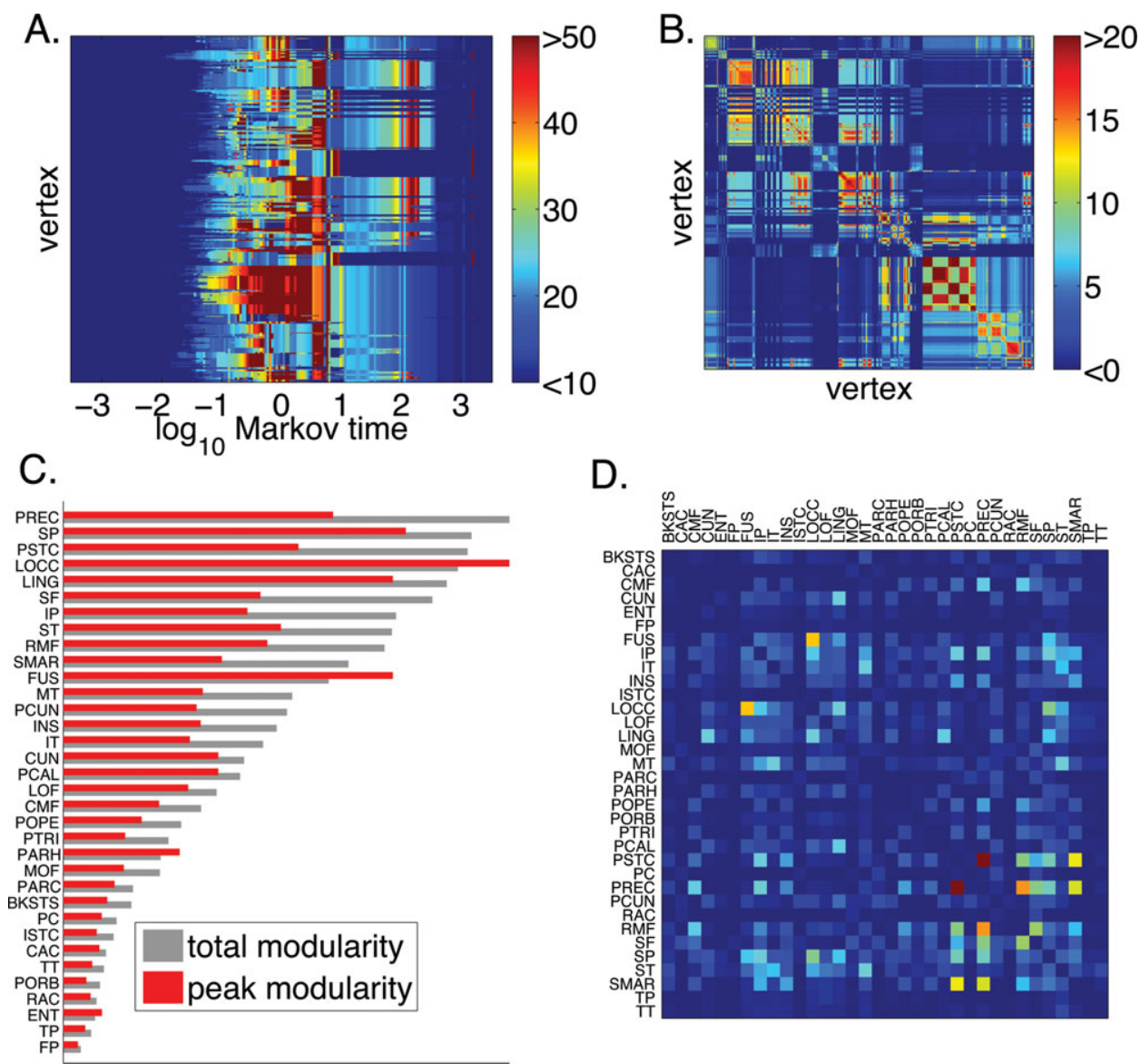

Fig. 7. Summary of modularity scores of communities at different times; (A) sum of positive and negative normalized scores by vertex over time; (B) weighted matrix where each community co-assignment was weighted by its score; $(\mathrm{C})$ anatomical area average and peak standardized modularity score; (D) weighted agreement matrix aggregated across by anatomical area. (Color online)

As a second means of relating community structure to observed functional connectivity, we measured the extent to which consensus communities were also good functional communities. This process consisted of estimating every consensus community's standardized modularity score. Over a range of scales from $t \approx 10^{-1.5}$ to $t \approx 10^{2.5}$, a number of communities had much greater-than-expected modularity (Figure 7(A)). Mapping these scores onto brain anatomy and summing across scales, it was observed that every cortical area contributed positive modularity, though some contributed disproportionately more (Figure 7(C)). The areas contributing the greatest modularity (both in terms of peak value and total contribution) were found to be the precentral and postcentral cortex, the lateral occipital cortex, the superior parietal cortex, and lingual cortex. Other areas also had large values, including the rostral middle-frontal cortex, superior frontal cortex, the inferior parietal cortex, as well as the superior temporal, supramarginal, and fusiform cortex.

The matrix $T_{i j}$ was constructed to measure the pairs of vertices that collectively participated in communities with greater-than-expected modularity (Figure 7(B)). 
A.
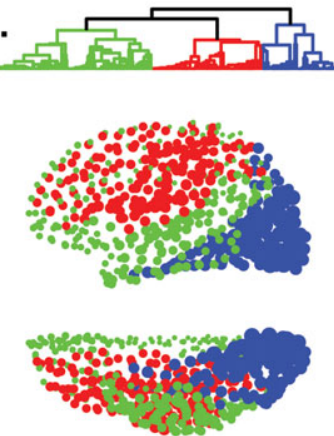

D.
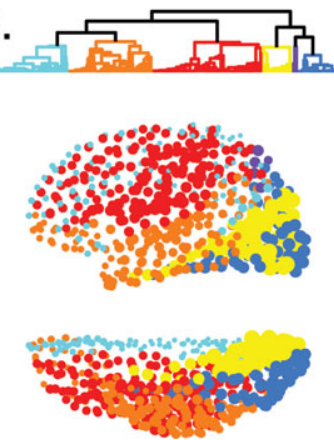

B.
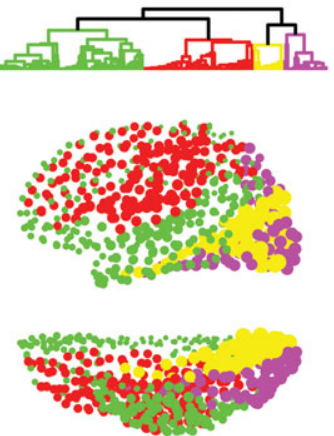

E.
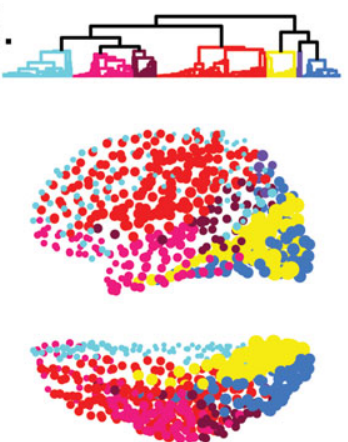

C.
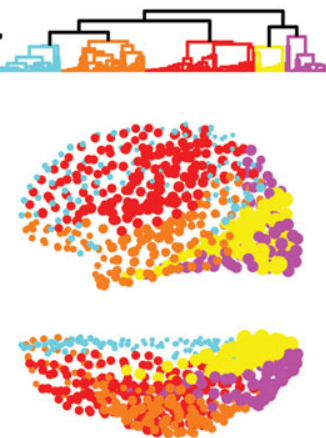

F.
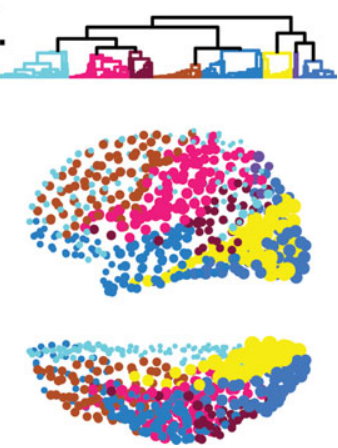

Fig. 8. Summary of hierarchical tree cut at different levels; (A)-(F) cuts reveal spatially contiguous maps of three to eight clusters. (Color online)

This matrix was mapped to the space of cortical areas by aggregating the values of vertex pairs that linked cortical areas. This process revealed a number of areas of cortex that, together, participated in communities with large modularity (Figure 7(D)). The largest contributing pairs were precentral/postcentral, lateral occipital/fusiform, supra-marginal/postcentral, supra-marginal/precentral, and rostral-middle-frontal/precentral cortices.

At the vertex level, $T_{i j}$ was clustered to reveal groups of vertices that collectively participated in communities with greater-than-expected modularity (Figure 8(A)(F)). Clustering produced a hierarchical tree, which was cut at different levels to reveal more or less clusters. At a coarse scale, $T_{i j}$ was decomposable into three spatially contiguous modules (Figure 8(A)): The first module (green) spanned most of the cortical midline including superior frontal and superior temporal cortex; the second module (red) was composed of both precentral and postcentral cortices as well as the rostral middle-frontal cortex; the third module (blue) consisted of lateral occipital, lingual, and superior parietal cortex, along with the fusiform gyrus.

Cutting the hierarchical tree at a lower level divided these macro-scale modules into smaller sub-modules. The first module (green) underwent a series of divisions into three smaller sub-modules (first split in Figure 8(C); second split in Figure 8(E)). The first split essentially divided frontal areas along the midline from the temporal cortex; the second split divided the temporal area from several parietal areas. The second module (red) survived largely unchanged across the range of clusters shown in Figure 8, before fragmenting as parts of pre- and post-central cortices formed a new cluster. The third module (blue) was also divided into, essentially, two 
sub-modules, the first made up predominantly by the lingual cortex, and the second made up of the fusiform area, lateral occipital, and superior parietal cortices. In principle, $T_{i j}$ could be decomposed further until the number of modules was equal to the number of vertices in the network.

\section{Discussion}

In this paper, we used the partition stability framework to infer multi-scale community structure in the human cerebral cortex. This procedure generated a series of communities over a range of scales, beginning with a large number of small communities (fine-scale) and ending with a small number of large communities (coarse-scale). We compared communities derived from structural connectivity to brain functional networks using two approaches: (i) We identified a scale at which the Pearson correlation of an agreement matrix with empirically measured rsFC became maximal, with a peak correlation of approximately $r=0.50$; (ii) We evaluated the modularity of each consensus community when it was imposed on rsFC, and identified a number of communities that overlapped with rsFC modules. These results suggest that a community's position in space and the scales over which it appears, provide complementary information for assessing its importance and relevance to network function.

Before discussing these results, it is worth explicitly stating our views of stability maximization and its relationship to the human connectome and communication processes in the brain. Communities derived from maximizing partition stability can be interpreted in multiple ways. The first interpretation is based on the view that stability maximization is simply a useful methodology for identifying community structure across multiple scales; no special functional significance is attributed to communities detected this way. The second interpretation regards such communities as being important to a dynamical process (e.g., diffusion), i.e. communities reflect the structural properties of a network that bias the trajectory of a random walker exploring the network. If the random walk is, in some way, a suitable model of the network's intrinsic dynamics, then stability-derived communities take on even more significance. In such a case, communities represent groups of nodes that more readily communicate or exert influence over one another than they do with the rest of the network. This paper adopts a view more closely aligned with the second interpretation. The argument for doing so stems from a conceptualization of the connectome as a communication network: Brain regions at different scales exert certain influence over one another, and this influence is subserved by the anatomical connections linking brain regions. This discussion asserts that it is, in part, the connection topology that prescribes a region's preference to functionally link to another region, or for a group of regions to become mutually coupled. Hence, from this point of view, identifying communities of vertices that are likely to influence or communicate with one another is of great practical interest and can possibly illuminate true functional dependencies. Nonetheless, it should be noted that the relationship between intrinsic brain dynamics and diffusion/random-walk processes remains in need of empirical testing.

The first major finding was that the correlation of the scale-dependent agreement matrix, which reflected the consistency of community ties between vertices at a 
given time point across all subjects, with rsFC reached a clear peak value. This result owes its significance to the interpretation of community structure as a marker of the propensity for vertices belonging to the same community to spread influence, information, or energy among one another and not with the rest of the network. Given this point of view, one can think of the peak correlation as corresponding to the scale at which empirical functional couplings became most closely aligned with influential communities that are consistently identified across subjects. This result is significant, as the functional coupling of different brain regions has been interpreted as integration of information (van den Heuvel \& Hulshoff Pol, 2010).

The scale at which the peak correlation occurred is also particularly relevant as it coincided with a regime characterized by partitions associated with large stability values but also many non-singleton communities. At much earlier scales, the average partition stability was slightly greater, but the number of singleton communities vastly outnumbered the non-singleton communities. At later scales, stability tended toward zero, so that even though the communities at those scales were much fewer in number and contained more vertices, they were also more ill-defined than those at earlier scales. This suggests that the peak correlation occurs within a fairly narrow regime in which the number and size of communities strikes a balance with stability, giving rise to a diverse, well-defined repertoire of communities.

The first result, however, only reveals part of the story. Linear correlation is a measure of how closely two variables correspond to one another on average. When examining the relationship between community structure and $\mathrm{rsFC}$ at the level of individual communities, a different (but complementary) picture emerges. Specifically, it was found that substantial variability existed in the timing of and extent to which individual communities contributed to the modularization of the rsFC matrix. Certain communities, even at scales where the partition as a whole was only weakly related to rsFC, had exceptionally large standardized modularity. At the time of the peak correlation, a large number of communities collectively had substantial modularity, which likely contributed to the peak in the correlation coefficient. This result, however, suggests that the relationship between rsFC and community structure is not restricted to a single scale.

A third interesting finding concerns the relationship of community structure to empirically observed resting state networks (RSN). The communities inferred here were generally spatially contiguous due to the fact that the majority of structural connections identified so far in the human connectome are short-distance and connect nearby regions to one another. Therefore, it was unsurprising that distributed and spatially non-contiguous RSNs, e.g., the right parietal-frontal and default mode network, were never observed. However, highly modular communities were found in occipital cortex, corresponding quite closely to the primary and extra-striate visual RSNs (lingual gyrus, cuneus, lateral occipital, fusiform). Also observed were high-modularity communities involving areas that are typically associated with the somatomotor network (pre- and postcentral cortex). Interestingly, both visual and somatomotor RSNs are generally classified as unimodal networks and thought to comprise tightly coupled and functionally related areas that jointly participate in sensorimotor processes (Bassett et al., 2013; Sepulcre et al., 2012). While the community structures that we observed do not directly correspond to the boundaries of these RSNs, their approximate resemblance is suggestive of a relationship between 
the dynamic process used to identify communities (random walk) and their strong functional couplings observed in the course of endogenously driven neural activity. Future work is needed to further clarify the nature of this relationship.

There are a number of limitations of this work that should also be discussed. Network studies of brain connectivity can be sensitive to parcellation schemes, which define network nodes and edges (Zalesky et al., 2010). While we did not systematically explore alternative parcellation schemes, coarser random parcellations yielded qualitatively similar results in our study. Another potential limitation concerns the nature of the random walk dynamics used to identify vertex communities. Random walks as a simple class of linear dynamics are limited in the types of behaviors they can exhibit (e.g., given that the network is connected and not bipartite, the random walk always evolves to a single stationary distribution) (Grinstead \& Snell, 1997). Other classes of dynamical systems, especially non-linear systems, can exhibit far more complex behaviors, including deterministic chaos and parameter sensitivity (bifurcations), among others. We defend our decision to use random walks on the grounds that the partition stability measure depends on this choice of dynamics to analytically define community structure. Furthermore, the well-documented behavior of a random walk drastically simplifies the analysis and interpretation of results.

Even when we restrict ourselves to the random walk class of dynamics, we still have room to elaborate our model further with the addition of parameters that bias the random walk, altering the transition preference or the rate at which random walkers leave vertices. These parameters may be defined on a vertex-wise basis and afford us means of artificially introducing heterogeneity into the random walk dynamics (Lambiotte et al., 2011). In this article, these parameters were set to fairly conservative values, i.e. a random walker's transition was made without bias and the rate at which transitions took place was equal for all vertices. Note that this parameterization is the most naïve we could have selected - choosing to bias the random walk or to imbue certain vertices with faster or slower transition rates would both require empirical justification.

\section{Conclusion}

This article aims to offer insight into the relationship between models of diffusion processes unfolding on the structural connectome, community structure, and empirically measured dynamic couplings. By maximizing partition stability, communities at multiple dynamical scales were identified. These communities were then compared to observed patterns of neural activity through a simple correlation measure and also by assessing each community's contribution to the modularization of rsFC. It was observed that a number of anatomical areas contributed disproportionately to this modularization, suggesting that those areas might be more functionally important than others. Future work is needed to further illuminate the role of different classes of dynamic processes in generating patterns of rsFC in complex brain networks.

\section{Acknowledgements}

Supported in part by: The National Science Foundation/IGERT Training Program in the Dynamics of Brain-Body-Environment Systems at Indiana University (RB); 
Swiss National Science Foundation, SNF grant 320030-130090 (AG); Spanish Government grant, contract number E-28-2012-0504681 (JG); Leenards Foundation, Switzerland (PH); JS McDonnell Foundation (OS and JG).

\section{Supplementary materials}

For supplementary material for this article, please visit http://dx.doi.org/10.1017/ nws.2013.19

\section{References}

Albert, R., Jeong, H., \& Barabási, A. L. (1999). Internet: Diameter of the world-wide web. Nature, 401(6749), 130-131.

Arenas, A., Fernandez, A., \& Gomez, S. (2008). Analysis of the structure of complex networks at different resolution levels. New Journal of Physics, 10(5), 053039.

Bassett, D. S., Greenfield, D. L., Meyer-Lindenberg, A., Weinberger, D. R., Moore, S. W., \& Bullmore, E. T. (2010). Efficient physical embedding of topologically complex information processing networks in brains and computer circuits. PLoS Computational Biology, 6(4), e1000748.

Bassett, D. S., Porter, M. A., Wymbs, N. F., Grafton, S. T., Carlson, J. M., \& Mucha, P. J. (2013). Robust detection of dynamic community structure in networks. Chaos, 23(1), 013142.

Bassett, D. S., Wymbs, N. F., Porter, M. A., Mucha, P. J., Carlson, J. M., \& Grafton, S. T. (2011). Dynamic reconfiguration of human brain networks during learning. Proceedings of the National Academy of Sciences USA, 108(18), 7641-7646.

Bassett, D. S., Wymbs, N. F., Rombach, M. P., Porter, M. A., Mucha, P. J., \& Grafton, S. T. (2013). Task-based core-periphery organization of human brain dynamics. PLoS Computational Biology, 9(9), e1003171.

Birn, R. M., Molloy, E. K., Parker, T., Meier, T. B., Kirk, G. R., Nair, V. A., ... Prabhakaran, V. (2013). The effect of scan length on the reliability of resting-state fMRI connectivity estimates. Neuroimage, 83, 550-558.

Blondel, V. D., Guillaume, J. L., Lambiotte, R., \& Lefebvre, E. (2008). Fast unfolding of communities in large networks. Journal of Statistical Mechanics: Theory and Experiment, 2008(10), P10008.

Bullmore, E., \& Sporns, O. (2009). Complex brain networks: Graph theoretical analysis of structural and functional systems. Nature Reviews Neuroscience, 10(3), 186-198.

Bullmore, E., \& Sporns, O. (2012). The economy of brain network organization. Nature Reviews Neuroscience, 13(5), 336-349.

Cammoun, L., Gigandet, X., Meskaldji, D., Thiran, J. P., Sporns, O., Do, K. Q., ... Hagmann, P. (2012). Mapping the human connectome at multiple scales with diffusion spectrum MRI. Journal of Neuroscience Methods, 203(2), 386-397.

Carpineto, C., \& Romano, G. (2012). Consensus clustering based on a new probabilistic rand index with application to subtopic retrieval. IEEE Transactions on Pattern Analysis and Machine Intelligence, 34(12), 2315-2326.

Chen, Z. J., He, Y., Rosa-Neto, P., Germann, J., \& Evans, A. C. (2008). Revealing modular architecture of human brain structural networks by using cortical thickness from MRI. Cerebral Cortex, 18(10), 2374-2381.

Chu, C. W., Holliday, J. D., \& Willett, P. (2012). Combining multiple classifications of chemical structures using consensus clustering. Bioorganic and Medical Chemistry, 20(18), 5366-5371. 
Daducci, A., Gerhard, S., Griffa, A., Lemkaddem, A., Cammoun, L., Gigandet, X., ... Thiran, J. P. (2012). The Connectome Mapper: An open-source processing pipeline to map connectomes with MRI. PloS One, 7(12), e48121.

Damoiseaux, J. S., Rombouts, S. A. R. B., Barkhof, F., Scheltens, P., Stam, C. J., Smith, S. M., \& Beckmann, C. F. (2006). Consistent resting-state networks across healthy subjects. Proceedings of the National Academy of Sciences, 103(37), 13848-13853.

Delmotte, A., Tate, E. W., Yaliraki, S. N., \& Barahona, M. (2011). Protein multi-scale organization through graph partitioning and robustness analysis: Application to the myosin-myosin light chain interaction. Physical Biology, 8(5), 055010.

Delvenne, J. C., Yaliraki, S. N., \& Barahona, M. (2010). Stability of graph communities across time scales. Proceedings of the National Academy of Sciences, 107(29), 12755-12760.

Fenn, D. J., Porter, M. A., McDonald, M., Williams, S., Johnson, N. F., \& Jones, N. S. (2009). Dynamic communities in multichannel data: An application to the foreign exchange market during the 2007-2008 credit crisis. Chaos, 19(3), 033119.

Fenn, D. J., Porter, M. A., Mucha, P. J., McDonald, M., Williams, S., Johnson, N. F., \& Jones, N. S. (2012). Dynamical clustering of exchange rates. Quantitative Finance, 12(10), $1493-1520$.

Flake, G. W., Lawrence, S., Giles, C. L., \& Coetzee, F. M. (2002). Self-organization and identification of web communities. Computer, 35(3), 66-70.

Fortunato, S. (2010). Community detection in graphs. Physics Reports, 486(3), 75-174.

Fortunato, S., \& Barthelemy, M. (2007). Resolution limit in community detection. Proceedings of the National Academy of Sciences, 104(1), 36-41.

Fox, M. D., \& Raichle, M. E. (2007). Spontaneous fluctuations in brain activity observed with functional magnetic resonance imaging. Nature Reviews Neuroscience, 8(9), 700-711.

Freeman, L. C. (2004). The development of social network analysis. Vancouver: Empirical Press.

Girvan, M., \& Newman, M. E. (2002). Community structure in social and biological networks. Proceedings of the National Academy of Sciences, 99(12), 7821-7826.

Good, B. H., de Montjoye, Y. A., \& Clauset, A. (2010). Performance of modularity maximization in practical contexts. Physical Review E, 81(4), 046106.

Gong, G., He, Y., Concha, L., Lebel, C., Gross, D. W., Evans, A. C., \& Beaulieu, C. (2009). Mapping anatomical connectivity patterns of human cerebral cortex using in vivo diffusion tensor imaging tractography. Cerebral Cortex, 19(3), 524-536.

Greicius, M. D., Krasnow, B., Reiss, A. L., \& Menon, V. (2003). Functional connectivity in the resting brain: A network analysis of the default mode hypothesis. Proceedings of the National Academy of Sciences, 100(1), 253-258.

Grinstead, C. C. M., \& Snell, J. L. (Eds.). (1997). Introduction to probability (2nd ed.). Providence, RI: American Mathematical Society.

Guimera, R., \& Amaral, L. A. N. (2005). Cartography of complex networks: Modules and universal roles. Journal of Statistical Mechanics: Theory and Experiment, 2005(02), P02001.

Hagmann, P., Cammoun, L., Gigandet, X., Meuli, R., Honey, C. J., Wedeen, V. J., \& Sporns, O. (2008). Mapping the structural core of human cerebral cortex. PLoS Biology, 6(7), e159.

Haimovici, A., Tagliazucchi, E., Balenzuela, P., \& Chialvo, D. R. (2013). Brain organization into resting state networks emerges from the connectome at criticality. Physical Review Letters, 110(17), 178101.

Hastie, T., Tibshirani, R., \& Friedman, J. J. H. (2001). The elements of statistical learning, Vol. 1. New York: Springer.

Honey, C. J., Sporns, O., Cammoun, L., Gigandet, X., Thiran, J. P., Meuli, R., \& Hagmann, P. (2009). Predicting human resting-state functional connectivity from structural connectivity. Proceedings of the National Academy of Sciences, 106(6), 2035-2040. 
Jonsson, P. F., Cavanna, T., Zicha, D., \& Bates, P. A. (2006). Cluster analysis of networks generated through homology: Automatic identification of important protein communities involved in cancer metastasis. BMC Bioinformatics, 7(1), 2.

Lambiotte, R. (2010). Multi-scale modularity in complex networks. In Modeling and optimization in mobile, ad hoc and wireless networks (WiOpt), 2010 Proceedings of the 8th International Symposium on (pp. 546-553). Avignon, France: IEEE.

Lambiotte, R., Delvenne, J. C., \& Barahona, M. (2008). Laplacian dynamics and multiscale modular structure in networks. arXiv preprint arXiv:0812.1770.

Lambiotte, R., Sinatra, R., Delvenne, J. C., Evans, T. S., Barahona, M., \& Latora, V. (2011). Flow graphs: Interweaving dynamics and structure. Physical Review E, 84(1), 017102.

Lancichinetti, A., \& Fortunato, S. (2012). Consensus clustering in complex networks. Scientific Reports, 2, 1-7.

Lewis, A. C. F., Jones, N. S., Porter, M. A., \& Deane, C. M. (2010). The function of communities in protein interaction networks at multiple scales. BMC Systems Biology, $\mathbf{4}$, 100.

Meunier, D., Lambiotte, R., \& Bullmore, E. T. (2010). Modular and hierarchically modular organization of brain networks. Frontiers in Neuroscience, 4, 200.

Moody, J., \& White, D. R. (2003). Structural cohesion and embeddedness: A hierarchical concept of social groups. American Sociological Review, 68(1), 103-127.

Mucha, P. J., Richardson, T., Macon, K., Porter, M. A., \& Onnela, J. P. (2010). Community structure in time-dependent, multiscale, and multiplex networks. Science, 328(5980), 876878.

Murphy, K., Birn, R. M., Handwerker, D. A., Jones, T. B., \& Bandettini, P. A. (2009). The impact of global signal regression on resting state correlations: Are anti-correlated networks introduced? Neuroimage, 44(3), 893-905.

Newman, M. E. (2006). Modularity and community structure in networks. Proceedings of the National Academy of Sciences, 103(23), 8577-8582.

Newman, M. E., \& Girvan, M. (2004). Finding and evaluating community structure in networks. Physical Review E, 69(2), 026113.

Onnela, J.-K., Fenn, D. J., Reid, S., Porter, M. A., Mucha, P. J., Fricker, M. D., \& Jones, N. S. (2012). Taxonomies of networks from community structure. Physical Review E, 86(3), 036104.

Power, J. D., Barnes, K. A., Snyder, A. Z., Schlaggar, B. L., \& Petersen, S. E. (2012). Spurious but systematic correlations in functional connectivity MRI networks arise from subject motion. Neuroimage, 59(3), 2142-2154.

Reichardt, J., \& Bornholdt, S. (2006). Statistical mechanics of community detection. Physical Review E, 74(1), 016110.

Ronhovde, P., \& Nussinov, Z. (2009). Multiresolution community detection for megascale networks by information-based replica correlations. Physical Review E, 80(1), 016109.

Rubinov, M., \& Sporns, O. (2011). Weight-conserving characterization of complex functional brain networks. Neuroimage, 56, 2068-2079.

Schaub, M. T., Delvenne, J. C., Yaliraki, S. N., \& Barahona, M. (2012). Markov dynamics as a zooming lens for multiscale community detection: Non clique-like communities and the field-of-view limit. PloS One, 7(2), e32210.

Sepulcre, J., Sabuncu, M. R., Yeo, T. B., Liu, H., \& Johnson, K. A. (2012). Stepwise connectivity of the modal cortex reveals the multimodal organization of the human brain. The Journal of Neuroscience, 32(31), 10649-10661.

Smith, S. M., Fox, P. T., Miller, K. L., Glahn, D. C., Fox, P. M., Mackay, C. E., ... Beckmann, C. F. (2009). Correspondence of the brain's functional architecture during activation and rest. Proceedings of the National Academy of Sciences, 106(31), 13040-13045. 
Sporns, O., Tononi, G., \& Kötter, R. (2005). The human connectome: A structural description of the human brain. PLoS Computational Biology, 1(4), e42.

Strehl, A., \& Ghosh, J. (2003). Cluster ensembles - a knowledge reuse framework for combining multiple partitions. The Journal of Machine Learning Research, 3, 583-617.

Topchy, A., Jain, A. K., \& Punch, W. (2005). Models of consensus and weak partitions. IEEE Transactions on Pattern Analysis and Machine Intelligence, 27(12), 1866-1881.

Traag, V. A., \& Bruggeman, J. (2009). Community detection in networks with positive and negative links. Physical Review E, 80(3), 036115.

van den Heuvel, M. P., \& Hulshoff Pol, H. E. (2010). Exploring the brain network: A review on resting-state fMRI functional connectivity. European Neuropsychopharmacology, 20(8), $519-534$.

van den Heuvel, M. P., Kahn, R. S., Goñi, J., \& Sporns, O. (2012). High-cost, high-capacity backbone for global brain communication. Proceedings of the National Academy of Sciences, 109(28), 11372-11377.

van den Heuvel, M. P., \& Sporns, O. (2011). Rich-club organization of the human connectome. The Journal of Neuroscience, 31(44), 15775-15786.

Vértes, P. E., Alexander-Block, A. F., Gogtay, N., Giedd, J. N., Rapoport, J. L., \& Bullmore, E. T. (2012). Simple models of human brain functional networks. Proceedings of the National Academy of Sciences, 109(15), 5868-5873.

Wedeen, V. J., Wang, R. P., Schmahmann, J. D., Benner, T., Tseng, W. Y. I., Dai, G., ... De Crespigny, A. J. (2008). Diffusion spectrum magnetic resonance imaging (DSI) tractography of crossing fibers. Neuroimage, 41(4), 1267-1277.

Wu, K., Taki, Y., Sato, K., Sassa, Y., Inoue, K., Goto, R., ... Fukuda, H. (2011). The overlapping community structure of structural brain network in young healthy individuals. PloS One, 6(5), e19608.

Yu, Z., Wong, H. S., \& Wang, H. (2007). Graph-based consensus clustering for class discovery from gene expression data. Bioinformatics, 23(21), 2888-2896.

Zalesky, A., Fornito, A., Harding, I. H., Cocchi, L., Yücel, M., Pantelis, C., \& Bullmore, E. T. (2010). Whole-brain anatomical networks: Does the choice of nodes matter? Neuroimage, 50(3), 970-983. 